\title{
PTCA OR RF ABLATION: WHICH IS TO BE DONE FIRST FOR PSVT ASSOCIATED WITH CAD
}

\author{
A Ashok Kumar, Ghanta Somasekhar
}

\section{INTRODUCTION:}

Supraventricular arrhythmias, other than atrial fibrillation or flutter, are relatively uncommon due to ischemia. Their occurrence often indicates myocardial dysfunction and they may, by themselves, cause congestive heart failure or exacerbate ongoing myocardial ischemia. The increase in the prevalence of SVT during an acute MI has been ascribed to atheromatous disease of the arteries supplying the atrioventricular (AV) node which is a rare cause. Ischemia of $\mathrm{AV}$ node results in in-homogeneity of the refractory period of AV nodal pathways and precipitating AVNRT[1]. Statin therapy, possibly due to an anti-inflammatory effect, has been associated with a reduction in SVT recurrences in patients with SVT, ischemic heart disease, and after cardiac bypass surgery. In a retrospective study of over 3300 patients presenting with acute myocardial infarction and in sinus rhythm, statin therapy (prescription within 48 hours of hospitalization) was associated with a reduced risk of SVT [2] predominantly AF (Atrial Fibrillation) (Odds Ratio $0.64,95 \%$ CI 0.45-0.92).

We report a case of CAD (coronary artery disease)of RCA and LAD presenting as PSVT and its recurrences are prevented after revascularization in its 6 months follow-up.

\section{CASE:}

A 62-year-old man, presented with chest pain and palpitation for2 hours at rest. He had similar episodes thrice in the past 6 months when the symptoms subsided spontaneously and the patient did not seek medical attention. PCI to RCA and RAMUS was done nine years back. He is hypertensive and non-diabetic. He had40-pack-year smoking and daily alcoholic drink history. On admission, his blood pressure was 130/90 $\mathrm{mm} \mathrm{Hg}$, pulse rate $>100$ beats per minute, respiratory rate 20 per minute and body temperature $36.6^{\circ} \mathrm{C}$. The electrocardiogram showed PSVT with pulse rate 190 bpm (Fig. 1).

Fig 1: ECG showing SVT

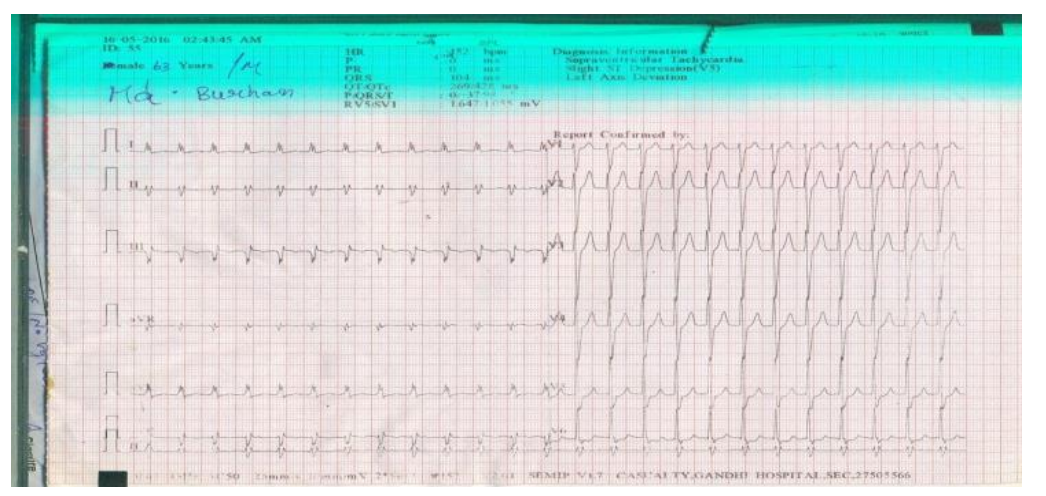

The patient has treated with adenosine $6 \mathrm{mg}$ twice and converted to sinus rhythm. Follow-up electrocardiography displayed no ST-T changes but frequent ectopics (Fig.2). Blood pressure was 150/100 $\mathrm{mm} \mathrm{Hg}$ with heart rate of $80 \mathrm{bpm}$. Cardiacenzyme showed transient increase; creatine phosphokinase -232 IU/L. Hemoglobin was $11.3 \mathrm{gm} / \mathrm{dl}$, TLC was 9100 cells/mm3, platelet count was 2.5 lakhs $/ \mathrm{mm} 3$, blood urea was $19 \mathrm{mg} / \mathrm{dl}$, serum creatinine was $1 \mathrm{mg} / \mathrm{dl}, \mathrm{Na}+$ was $136 \mathrm{mmoles} / \mathrm{L}, \mathrm{K}+$ was $5.2 \mathrm{mmoles} / \mathrm{L}$, ESR was 5 $\mathrm{mm}$. He had no other abnormal laboratory findings. Echocardiogram exhibited preserved left ventricle(LV) systolic function and concentric LV hypertrophy. But mild hypo-kinesia in septum and anterior wall of LV was noted. 
Fig 2 : Post adenosine ECG with normal sinus rhythm

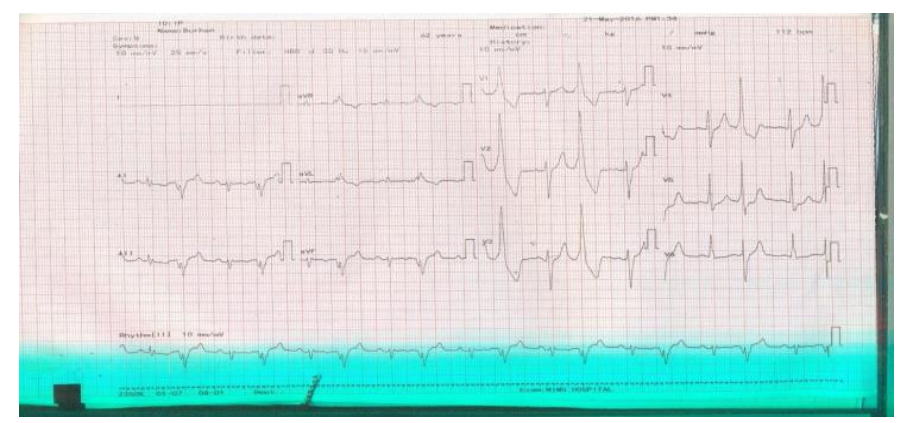

He was diagnosed with PSVT and temporary acute myocardial injury. After the acute episode terminated, he was treated with aspirin, lipid lowering agent, angiotensin II receptor blocker and intravenous heparin.24 hours holter monitoring showed one episode of non-sustained PCVT for8 seconds. Coronary angiography revealed the significant CAD in LAD and RCA (Fig3, Fig 4).

Fig 3: CAG showing lesion in mid LAD.

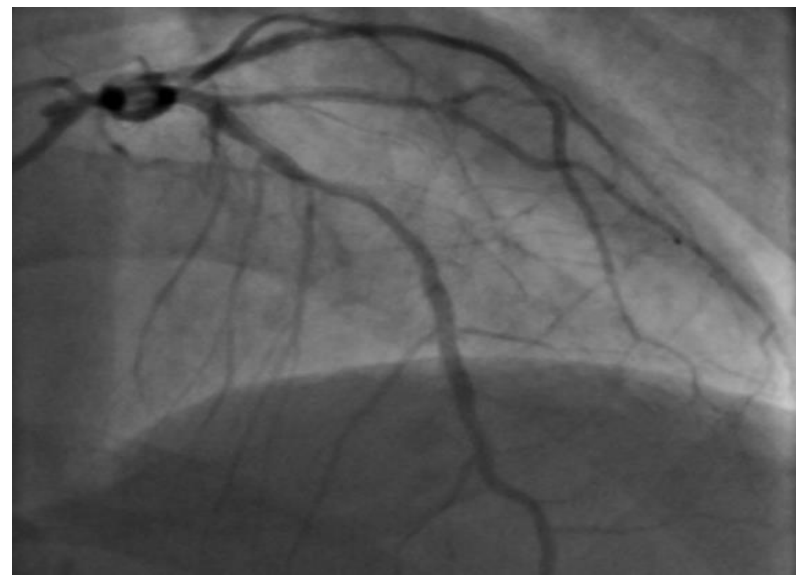

Fig 4: CAG showing lesion in mid-RCA

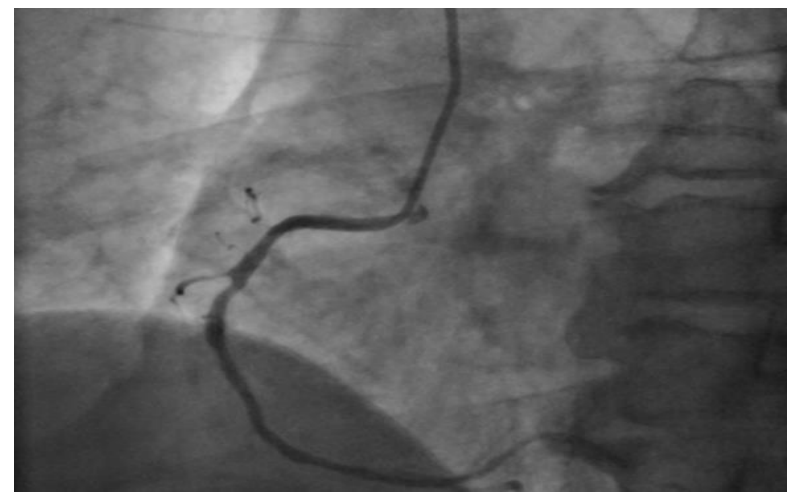

Successful PCI done to LAD and RCA (Fig 5, Fig 6). We continued medical treatment including ATII receptor blocker, metoprolol and DAPT and recommended lifestyle modification. During the follow-up of one year there were no episodes of SVT.

Fig 5: Post PTCA angiogram to mid LAD

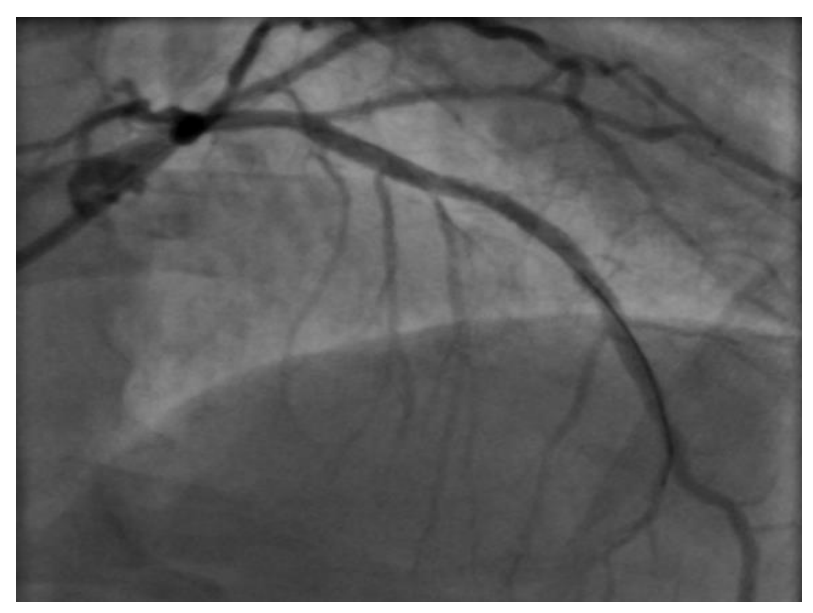

Fig 6: Post PTCA angio to mid-RCA

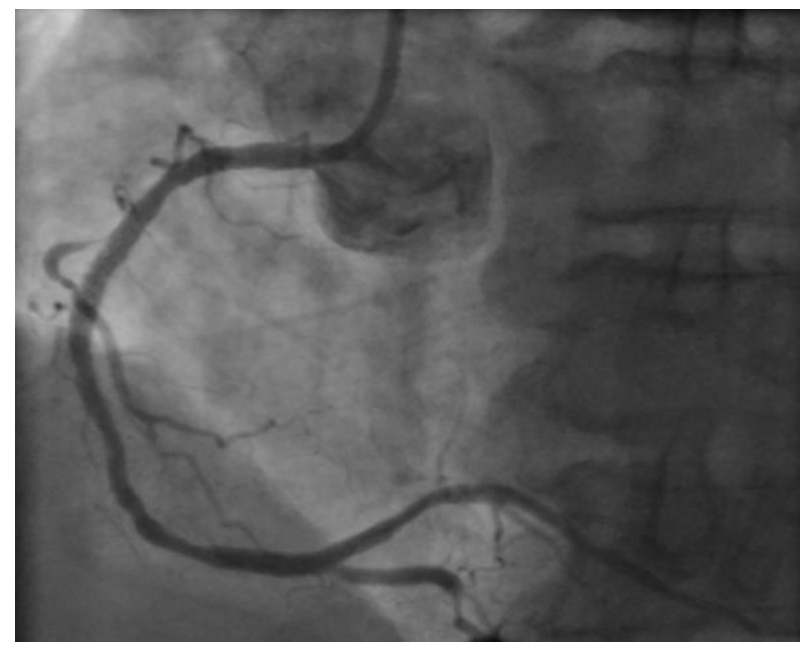

\section{DISCUSSION:}

\section{ROLE OF PTCA:}

The natural history of SVT is steadily changing because most patients with SVT undergo ablation at a younger age, but in general, the relative proportion of AT is higher in older populations, and AVNRT is more prevalent than AVRT among patients undergoing ablation [3]. There are limited outcome data from RCTs 
for an elderly segment of the population. Rising incidence of coronary artery disease in the recent times and increasing prevalence with age necessitates subjecting the patient to coronary angiogram(CAG) prior to EP study and RF ablation.

The association of tachy-arrhythmia and coronary ischemia is known but definite explanations are lacking. Some of the proposed mechanisms are dispersion in refractory period of longitudinal $\mathrm{AV}$ node fibers due $\mathrm{AV}$ nodal ischemia creating dual AV nodal physiology and consequently AVNRT and alteration in refractory period due to ischemia driven sympathetic surge precipitating reentry in existing dual AV node pathway.

Coronary artery disease has been associated with myocardialischemia, arrhythmia, and sudden death during exercise. The prevalence of PSVT due to ischemia has been reported between

0.6 to $1.3 \%$ in angiographic series [4]. It is believed that sudden arrhythmias such as PSVT are brought about by hemodynamic changes secondary to coronary artery disease. As hemodynamic embarrassment occurs with coronary artery disease and PSVT further compromising the hemodynamic necessitates PTCA to be subjected if CAD is identified through CAG prior to ablation and necessity of ablation considered in the follow-up if significant recurrences of PSVT occurs [5].

It is a limitation of this case report that the cause of PSVT, whether atrioventricular nodal reentry or accessory pathway, was not evaluated by cardiac electrophysiologic study. The electrophysiologic study could help to clarify the relation between coronary artery disease and PSVT.

\section{ABLATION:}

Data have consistently demonstrated that ablation is highly successful $(>95 \%)$ in selected older patients. Outcomes from 48 medical centers in Germany were reported among 3,234 consecutive patients undergoing AVNRT ablation from 2007 to 2010; of the total, 259 patients $(8 \%)$ were $>75$ years of age. Acute success was achieved in $98.5 \%$ of the older patient group, similar to the 2 younger patient groups $(98.7 \%$ for the group $<50$ years of age; $98.8 \%$ for the group 50 to 75 years of age)
[6]. In this study, complication rates were low; hemodynamically stable pericardial effusion was observed in 2 of 259 patients $(0.8 \%)$, and no pacemakers were needed in the older patient group [7]. Similarly, additional studies from older patient cohorts have consistently shown that older patients have more comorbid medical conditions, have a higher incidence of structural heart disease or ischemic heart disease, and have more severe symptoms associated with SVT. A few studies have shown that complications may be slightly higher in older patients than in younger patients, although the overall complications are low and acceptable [8]. These ablation outcome data should be balanced with the risks and benefits of pharmacological therapy when therapeutic options are reviewed with older patients [9].

\section{CONCLUSION:}

It is important that the patient be included in clinical decision-making processes, with consideration of his/her preferences and goals for therapy, as well as his/her unique physical, psychological, and social situation. In selected cases, personalized, self-directed interventions can be developed in partnership with the patient, such as vagal maneuvers and "pill-in-the-pocket" drug therapy. Shared decision making is especially important for patients with SVT and CAD. As seen in the guideline, SVT treatment can be nuanced and requires expert knowledge of EP processes and treatment options like PTCA [10]. Treatment options are highly specific to the exact type of arrhythmia and can depend on certain characteristics of a particular patient like associated CAD. The various choices for therapy, including drugs, cardioversion, invasive treatment with RF ablation, PTCA if CAD is associated and contributing or a combination thereof, can be confusing to the patient, so a detailed explanation of the benefits and risks must be included in the conversation. Patients are encouraged to ask questions with time allotted for caregivers to respond. There should be clear explanation of the risks and benefits of each recommendation, including how other comorbidities may impact each treatment option. It is the responsibility of the physician and healthcare team to provide the patient with the best possible understanding of all management options in terms of risks, benefits, and potential effects on quality of life. 


\section{REFERENCES:}

1.James TN. Myocardial infarction and atrial 7.Haghjoo M, Arya A, Heidari A, et al. arrhythmias. Circulation 1961; 24:761. Electrophysiological characteristics and results of radiofrequency catheter ablation in elderly patients with

2.Zimet baum PJ, Josephson ME. Use of the atrioventricular nodal reentrant tachycardia. J electrocardiogram in acute myocardial infarction. $\mathrm{N}$ Engl J Med 2003; 348:933.

Electrocardiol. 2007;40:208-13

8.Meissner A, Stifoudi I, Weismüller P, et al. Sustained

3.Berisso MZ, Ferroni A, Molini D, Vecchio C. [Supraventricular tachyarrhythmias during acute myocardial infarction: short- and mid-term clinical significance, therapy and prevention of relapse]. G ItalCardiol 1991; 21:49.

4.Ganz LI, Friedman PL. Supraventricular tachycardia. N Engl J Med 1995; 332:162.

5.Porter MJ, Morton JB, Denman R, et al. Influence of age and gender on the mechanism of supraventricular tachycardia. Heart Rhythm. 2004;1:393-6.

6.Razavi M, Luria DM, Jahangir A, et al. Acute blood pressure changes after the onset of atrioventricular nodal reentrant tachycardia: a time-course analysis. J Cardiovasc Electrophysiol. 2005;16: 1037-40.

high quality of life in a 5-year long term follow up after successful ablation for supra-ventricular tachycardia: results from a large retrospective patient cohort. Int J Med Sci. 2009;6:28-36.

9.Wood KA, Stewart AL, Drew BJ, et al. Patient perception of symptoms and quality of life following ablation in patients with supraventricular tachycardia. Heart Lung. 2010;39:12-20.

10.Yildirim O, Yontar OC, Semiz M, et al. The effect of radiofrequency ablation treatment on quality of life and anxiety in patients with supraventricular tachycardia. Eur Rev Med Pharmacol Sci. 2012;16: 2108-12. 